\title{
Análisis de Nutrientes de la Raíz de la Malanga (Colocasia esculenta Schott) en el Trópico Seco de Colombia
}

\author{
Amparo L. Púa(1), Genisberto E. Barreto(2), José L. Zuleta(2) y Oscar D. Herrera(2) \\ (1) Facultad de Nutrición y Dietética, Universidad del Atlántico, Calle 30 \#8-49 Puerto Colombia, Colombia. \\ (e-mail: amparopua@mail.uniatlantico.edu.co) \\ (2) Facultad de Química y Farmacia, Universidad del Atlántico, Calle 30 \#8-49 Puerto Colombia, Colombia. \\ (e-mail: genisbertobarreto@mail.uniatlantico.edu.co; jose.921@hotmail.com; oscarhc91@outlook.com)
}

Recibido Sep. 26, 2018; Aceptado Dic. 10, 2018; Versión final Mar. 2, 2019, Publicado Ago. 2019

\section{Resumen}

El objetivo del estudio fue analizar el contenido de nutrientes de la malanga cultivada en la zona rural del Caribe colombiano. A muestras de malanga se le realizaron análisis proximales, de vitaminas y minerales, con el empleo de los métodos Kjeldahl, Soxhlet, hidrólisis ácido-base, incineración, cromatografía y absorción atómica. Los resultados se compararon con el contenido de nutrientes del camote, la papa, la yuca y el ñame. Los resultados obtenidos fueron $24.99 \%$ de proteínas, $0.53 \%$ de grasa, $59.36 \%$ de carbohidratos, $7.65 \%$ de ceniza, $4.88 \%$ de fibra bruta, $2.59 \%$ de humedad, $8.35 \mathrm{mg} / \mathrm{kg}$ de hierro, $94.45 \mathrm{mg} / \mathrm{kg}$ de calcio, 242.37 $\mathrm{mg} / \mathrm{kg}$ de magnesio, $6.21 \mathrm{mg} / \mathrm{kg}$ de zinc, $0.188 \mathrm{mg} / 100 \mathrm{mg}$ de vitamina C, $0.047 \mathrm{mg} / 100 \mathrm{mg}$ de Vitamina B1, y $0.078 \mathrm{mg} / 100 \mathrm{mg}$ de Vitamina B3. El contenido de proteínas y grasas fue superior al de los otros vegetales comparados.

Palabras clave: composición química; carbohidratos; minerales; cromatografía; espectrofotometría

\section{Nutrient Analysis of Taro Root (Colocasia esculenta Schott) in the Dry Tropics of Colombia}

\begin{abstract}
The aim of the study was to analyze the nutrient content of the taro cultivated in rural areas of the Colombian Caribbean. To the samples of taro, proximal analyzes of vitamins and minerals were carried out, using the Kjeldahl, Soxhlet methods, acid-base hydrolysis, incineration, chromatography and atomic absorption. The results were compared with the nutrient content of sweet potato, potatoes, cassava and yams. The results obtained were $24.99 \%$ protein, $0.53 \%$ fat, $59.36 \%$ carbohydrate, $7.65 \%$ ash, $4.88 \%$ crude fiber, $2.59 \%$ moisture, $8.35 \mathrm{mg} / \mathrm{kg}$ of iron $94.45 \mathrm{mg} / \mathrm{kg}$ calcium $242.37 \mathrm{mg} / \mathrm{kg}$ magnesium, $6.21 \mathrm{mg} / \mathrm{kg}$ of zinc, 0.188 $\mathrm{mg} / 100 \mathrm{mg}$ of vitamin C, $0.047 \mathrm{mg} / 100 \mathrm{mg}$ of vitamin B1, and $0.078 \mathrm{mg} / 100 \mathrm{mg}$ vitamin B3. The content of proteins and fats was higher than the other vegetables compared.
\end{abstract}




\section{INTRODUCCIÓN}

La malanga es una planta herbácea suculenta, que alcanza una altura de 1-3 $\mathrm{m}$, sin tallo aéreo, también conocida como Ocumo chino, Taro o Dashen, es considerada una de las especies de raíces y tubérculos con gran potencial en las zonas tropicales. Su cultivo probablemente se originó en el norte de Suramérica, se extendió por las Antillas y Mesoamérica, se encuentra difundido desde los trópicos hasta los límites de las regiones templadas, necesita precipitaciones altas de 1800 a $2500 \mathrm{~mm}$, temperaturas entre $25^{\circ} \mathrm{C}$ y $35^{\circ} \mathrm{C}$ y buena luminosidad, se considera una planta tropical, que crece preferiblemente en zonas pantanosas y arcillosas, pero con abundante agua durante la cosecha (Viloria y Córdova, 2008). Pertenece a la familia Araceae y es una planta herbácea perenne que se cultiva anual (Lewu et al., 2009). Se considera que existen numerosas variedades botánicas de malanga, sin embargo, se han dividido dos grandes grupos: la tipo Dasheen, en la que el cormo central es grande y los cornelos son pequeños y la tipo Eddoe, en la que el cormo central es pequeño y los cornelos son grandes (Sefa-Dedeh y Agyir-Sackey, 2004).

Con relación a los usos de la malanga, Abdulrashid y Nnabuenyi (2009) utilizaron la malanga como alimento para aves de corral, para tal fin emplearon tratamientos dietéticos que contenían $0 \%, 25 \%, 50 \%$ y $100 \%$ de malanga en dos formas: en estado crudo y cocido, este se le administró a un grupo de aves y determinaron que la malanga cocida puede reemplazar hasta en un $50 \%$ el maíz en las dietas de engorde, sin embargo la malanga cruda, solo podía constituir el $25 \%$, con el fin de no alterar la carga nutricional requerida. Nwokocha et al., 2008, realizaron un estudio comparativo de algunas propiedades a nivel microscópico, térmico, físico.químico y reológico del almidón de la mandioca, con el almidón de la malanga, destacaron características favorables para la utilización del almidón de la malanga, tales como, mayor contenido de amilosa, alta gelatinización endotérmica y mayor resistencia a las condiciones de procesamiento, lo que los llevó a concluir que los almidones de estos alimentos, tienen muchas aplicaciones en la industria alimentaria. La malanga también ha sido utilizada en la elaboración de harinas compuestas, al igual que las harinas de cereales, leguminosas y frutos secos, la industria alimentaria las emplea en la elaboración de salsas, cremas, fideos, pastas; no obstante, la utilización de estas harinas depende de su composición y propiedades funcionales, que se relacionan con la fuente y las condiciones de cultivo, Rodríguez-Miranda et al., 2011.Torres et al., 2014, evaluaron el efecto de la sustitución de harina de trigo por almidón de malanga de las variedades blanca y morada, sobre las pérdidas por cocción y aceptabilidad de una emulsión cárnica y determinaron, que es posible reemplazar la harina de trigo utilizada como sustancia de relleno, por almidón de malanga de las dos variedades, para disminuir las perdidas por cocción en salchichas tipo Frankfurt, sin afectaciones negativas en la aceptabilidad.

En lo referente a las propiedades nutricionales de la malanga, el tallo central es elipsoidal, conocido como cormo (tallo subterráneo), este es alto en carbohidratos (18\%-30\% en base fresca) y en proteínas (1.7\%-2.5\% en base fresca) con respecto a otros vegetales de elevado consumo en el Caribe colombiano, como la papa. Sefa-Dedeh y Agyir-Sackey, 2004 estudiaron el efecto de los tratamientos de secado al horno y secado al sol, en las cornelos de dos especies de malanga, Xanthosoma sagittifolium y cornelos de Colocasia esculenta, en las secciones distal, media y apical y el contenido de oxalato de estos, encontraron 2.98-5.50 g/100 g de proteína cruda, 0.28-0.97 g/100 g de grasa total, 1.56-2.98 g/100 g de cenizas, $12.2-36 \mathrm{~g} / 100 \mathrm{~g}$ de almidón y $1.11-3.00 \mathrm{~g} / 100 \mathrm{~g}$ de fibra cruda, a su vez identificaron que la variedad de malanga Xanthosoma spp (variedad blanca-carne) tuvo el mayor valor nutritivo. Kaushal et al., 2012 compararon las propiedades físicoquímicas, funcionales y antinutricionales de la harina de malanga, con harinas de arroz y de pigeonpea y encontraron diferencias significativas, por el alto contenido de cenizas y fibra cruda de la malanga, pero bajo en grasas y proteínas; igualmente la harina de malanga presentó baja capacidad de absorción de espuma, alto índice de solubilidad en agua, alta capacidad de absorción de agua y de aceite, igualmente se observó que al aumentar el contenido de harina de malanga en las mezclas, se obtuvieron cambios positivos; su contenido de oxalatos y polifenoles es elevado y su contenido de fitatos es bajo. Alcantara et al., 2013, también estudiaron el valor nutricional y los componentes fitoquímicos y sustancias antinutricionales de la malanga (Colocasia esculenta (L.) Schott) en polvo y algunos alimentos procesados, seleccionados en Filipinas y determinaron que el procesamiento del cormo de la malanga afecta significativamente su composición proximal, contenido de minerales, componentes fitoquímicos y antinutricionales como los oxalatos y los fitatos, esto se observó cuando se obtuvo el polvo, tallarines y galletas, lo que podría deberse al empleo de altas temperaturas.

Ezeabara et al., 2015, investigaron cinco variedades de cormos de Colocasia esculenta (L.) Schott, encontradas en Estado de Anambra, sudeste de Nigeria: Colocasia esculentavar. antiquorum (L.) (Schott) Hubbard \&Rehder (eddoe), C. esculentavar. esculenta (L.) Schott (dasheen), 'Kochuo' - tubérculos púrpuras, 'Nwine' - tubérculos largos y 'Ogeriobosi' - corcho de cabeza grande. Se cree que los hallazgos de este estudio alientan el nivel de consumo de cormelos de estas variedades de Colocasia esculenta debido a sus altos valores nutricionales y medicinales, atribuídos al contenido de carbohidratos, proteínas, vitaminas $\mathrm{A}$ y C, calcio, fósforo y potasio, así como también al contenido de metabolitos activos. El antinutriente presente, 
el oxalato, no genera ningún problema nutricional, porque $C$. esculenta normalmente se somete a una cocción prolongada antes del consumo que, a su vez, eliminará los antinutrientes. Además, las variedades de $C$. esculenta son potencialmente el mejor cultivo para bebés, ancianos y pacientes diabéticos. Eleazu, 2016, investigó las propiedades antioxidantes de $C$. esculenta y reportó los productos naturales, que justifican sus propiedades biológicas, tales como el ácido 9-octadecenoico, con efectos antiprostáticos y propiedades hipocolesterolémicas.

Sobre el estado nutricional de la población colombiana, la Encuesta Nacional de la Situación Nutricional (ENSIN) 2010, en Colombia, reportó que uno de cada 6 niños de 1 a 4 años y 1 de cada 6 mujeres embarazadas presenta anemia; esta proporción es del $11 \%$ en jóvenes entre 13 y 17 años; el $8 \%$ de los niños de 5 a 12 años y 7,6\% de las mujeres en edad fértil, presentan anemia, estas cifras configuran un problema de salud pública con grado de severidad leve, según la Organización Mundial de la Salud (OMS). En general, la anemia se presenta en mayor proporción en el área rural y en la población clasificada con niveles 1 y 2 del Sistema de Información de Potenciales beneficiarios para Programas Sociales (SISBEN). Las regiones más afectadas con anemia son, Pacífica en menores de 5 años, Oriental en niños de 5 a 12 años y mujeres en edad fértil, y Atlántica en mujeres gestantes; uno de cada cuatro niños de 1 a 4 años presentó deficiencia de vitamina $A$; los niños de 1 a 2 años de la región Caribe, perteneciente al nivel 1 del SISBEN y que residen en el área rural; y cerca de 1 de cada 2 en este grupo de edad, presenta deficiencia de zinc, situación que se considera como un problema de salud pública (Profamilia et al., 2010).

Después de haber realizado una revisión bibliográfica y búsqueda de artículos científicos en diferentes base de datos sobre el contenido nutricional de la malanga, se observó poca información al respecto, la divulgación de sus propiedades, puede contribuir al aumento del consumo de este alimento y a la mejora de la situacional nutricional de la población, teniendo en cuenta que Colombia produce diversos tipos de raíces y tubérculos, como la malanga, sin embargo está sujeta a perdidas postcosecha importantes, tales como, mal estado de las vías y deficiente tecnificación durante esta etapa, lo que ocasiona que su uso y consumo sean limitados (Estrada et al., 2010). La región Caribe colombiana cuenta con unas condiciones adecuadas que permiten el crecimiento y desarrollo del cultivo de malanga, pero se hace necesario nuevos estudios para lograr mejorar el procesamiento y aprovechamiento de este vegetal. Los conocimientos disponibles sobre los componentes químicos más importantes, las características físicas, nutricionales y funcionales, influyen en las opciones para estimular el uso y consumo de las raíces y tubérculos andinos (Barrera et al., 2004). Con base en los valores nutricionales de la malanga reportado en otros países, las deficiencias de micronutrientes de la población colombiana y la escases de estudios sobre la malanga en Colombia, se desarrolló el presente estudio, con el objetivo de analizar el contenido de nutrientes de la malanga, cultivada en la zona rural de la región Caribe colombiana.

\section{MATERIALES Y MÉTODOS}

El estudio es de carácter descriptivo y el diseño de la investigación es de tipo prospectivo y de corte transversal.

\section{Selección del material vegetal}

La materia prima fue recolectada en la zona rural del municipio de Ovejas, altitud 254 m.s.n.m., 9 ${ }^{\circ} 31$ '33' $\mathrm{N}$ $75^{\circ} 13^{\prime} 38^{\prime} \mathrm{O}$, departamento de Sucre (Colombia), se seleccionó material vegetal con características de frescura. El área de muestreo presentó una temperatura de $34^{\circ} \mathrm{C}$.

\section{Obtención de la muestra}

Se empleó un muestreo por conveniencia, se seleccionó una muestra de $2 \mathrm{~kg}$ de malanga. El tamaño del campo muestreado fue de media hectárea, la fertilización recibida fue abono orgánico, se utilizó malanga larga, la puvliometría fue de 1600 a $1800 \mathrm{ml} / a n ̃ o$, en este tipo de cultivos el número de riegos se realiza de acuerdo a las condiciones climatológicas, la edad de la planta al momento del muestreo fue de 7 meses.

\section{Limpieza}

La malanga se sometió a lavado con agua tratada, para retirar cualquier material extraño (partículas de polvo, tierra y restos vegetales) que pudieran estar presentes en esta.

\section{Secado}

Se expuso el material vegetal bajo la luz directa del sol durante una hora, en el lapso de dos días. El tercer día se sometió a secado en una estufa de desecación marca Memert, durante $24 \mathrm{~h}$ a una temperatura de 100 ${ }^{\circ} \mathrm{C}$ (Pinzón y Cardona, 2008). 


\section{Molienda y pulverizado}

Se utilizó un molino eléctrico marca Universal, para disminuir el tamaño de partículas de la malanga y así obtener la muestra, utilizando un tamiz con mallas de $3 \mathrm{~mm}, 177$ y $100 \mu \mathrm{m}$ en su orden respectivamente, con el objetivo de obtener tamaños de partículas semejantes logrando un mezclado homogéneo.

\section{Análisis proximal}

La determinación de proteínas totales se basó en la destrucción de la materia orgánica con ácido sulfúrico concentrado, formándose sulfato de amonio que en exceso de hidróxido de sodio libera amoniaco, el que se destila recibiéndolo en ácido bórico, dando origen a borato de amonio, que se valora con ácido Sulfúrico 0.1 $\mathrm{N}$ y utilizando como indicador, Tashiro (AOAC, 2005). Se calculó en porcentaje de proteína, respecto al peso de la muestra seco según la ecuación establecida en el método de referencia (Ecuación 1):

$$
\text { Porcentaje de nitrógeno }=\text { V. de ácido } \times \text { N. de ácido } \times \text { mEqN/Wm } \times 100
$$

Dónde: N: Normalidad del ácido de valoración; V: Volumen de ácido consumido; $\mathrm{mEq} \mathrm{N}$ = miliequivalentes de nitrógeno; y $\mathrm{Wm}=$ peso de la muestra

La determinación de la fibra bruta se fundamentó en la digestión secuencial de la muestra sin grasa, con una solución de ácido sulfúrico $1.25 \% \mathrm{~m} / \mathrm{v}$, y con una solución de hidróxido de sodio $2.5 \% \mathrm{~m} / \mathrm{v}$, el residuo insoluble se colectó por filtración, se lavó, se secó, se taró y se llevó a una mufla Terrígeno, para corregir la contaminación por minerales (ICONTEC, 2001). Se calculó el porcentaje de fibra según el método de referencia (Ecuación 2):

$$
\text { Fibra bruta }=\mathrm{W}(\mathrm{g}) \text { crisol residuo seco }-\mathrm{W}(\mathrm{g}) \text { crisol vacío } \mathrm{W}(\mathrm{g}) \text { muestra } \times 100
$$

La determinación de cenizas se basó en la destrucción de la materia orgánica presente en la muestra, por calcinación y determinación gravimétrica del residuo (Pearson, 2013). Se calculó el porcentaje de cenizas, respecto al peso de muestra seca (Ecuación 3):

$$
\text { Cenizas }(\%)=(P 2-P 1) / M \times 100
$$

Dónde: $\mathrm{P} 1=$ Peso del crisol de porcelana vacío, $\mathrm{P} 2=\mathrm{Peso}$ del crisol de porcelana más cenizas y $\mathrm{M}=\mathrm{Pes}$ de la muestra inicial. La extracción de grasa se obtuvo con el empleo del disolvente n-hexano y se determinó gravimétricamente, después de la evaporación del disolvente (ICONTEC, 2000). El resultado fue expresado en \% de grasa (Ecuación 4):

$$
\% \text { de grasa }=\mathrm{W} 2-\mathrm{W} 1 / \mathrm{W} \text { muestra } \times 100
$$

La determinación de humedad se obtuvo tarando la muestra, posteriormente fue sometida a secado a $105^{\circ} \mathrm{C}$, se dejó enfriar y se taró nuevamente, para esto se utilizó el equipo analizador de humedad MB35 de halógeno (ICONTEC, 2009).

Para la determinación de carbohidratos, se tomaron los resultados obtenidos de los análisis de proteínas, grasas, humedad y cenizas de cada una de las muestras y se calculó el extracto nitrogenado (AOAC, 2005).

\section{Determinación de vitaminas y minerales}

Para la determinación de vitaminas, las condiciones cromatográficas fueron, fase móvil: Acido perclórico 0.1 $\mathrm{N}$-Acetonitrilo (8:1), Flujo: $0.4 \mathrm{~mL} / \mathrm{min}$, Volumen de inyección: $20 \mu \mathrm{L}$, Detector: $254 \mathrm{~nm}$. Para la preparación de la muestra, se tararon aproximadamente $5 \mathrm{~g}$ de muestra en un beacker de $250 \mathrm{~mL}$, se adicionaron $20 \mathrm{~mL}$ de ácido clohídrico $0.1 \mathrm{~N}$, luego se colocó en agitación aproximadamente 30 minutos, se adicionaron $0.5 \mathrm{~mL}$ de karrit y $0.5 \mathrm{~mL}$ de karrit 2, se mezcló y se filtró. Para la preparación del estándar, se transfirió de manera exacta, $50 \mathrm{mg}$ del estándar de cada vitamina a estudiar (niacina, tiamina, riboflavina y vitamina $\mathrm{C}$ ), a una volumétrica de $50 \mathrm{~mL}$, se agregaron $25 \mathrm{~mL}$ de ácido clorhídrico 0.01 y se agitó manualmente hasta disolver, se aforó y se mezcló. Finalmente se inyectó el estándar y la muestra en las mismas condiciones (Pharmabooks, 2005).

Para el análisis de minerales, se empleó el método de espectrofotometría de absorción atómica, que transforma la muestra de átomos, en estado de vapor y mide la absorción electromagnética emitida o absorbida por dichos átomos (Pharmabooks, 2005). Se realizó curva de calibración, utilizando concentraciones conocidas de los minerales previamente, para garantizar la reproducibilidad del método, para 
esto se empleó un estándar de solución a 100 ppm de cada uno de los minerales. Cada análisis tuvo una duración de 20 min aproximadamente. Los resultados obtenidos se expresaron en mg de cada mineral, por $\mathrm{g}$ de muestra seca.

Finalmente, los resultados del contenido de nutrientes de la malanga, se compararon de manera descriptiva con el contenido de nutrientes del camote papa, yuca, ñame, reportado en la Tabla de Composición química de Alimentos Colombianos (Instituto Colombiano de Bienestar Familiar 2015), para establecer semejanzas y diferencias, estos vegetales crecen bajo condiciones y tipo de suelos similares a las de la malanga. Para el análisis de los datos utilizaron herramientas estadísticas tales como: medidas de centralización, medidas de dispersión (S, S², RSD), intervalos de confianza y resultados anómalos: la prueba de Dixon (Mongay, 2011).

\section{RESULTADOS Y DISCUSIÓN}

La tabla 1 muestra la composición proximal de la malanga, los resultados demuestran que los carbohidratos son los nutrientes que se encuentran en mayor cantidad con un $59.359 \%(0.194)$, característico de las raíces y los tubérculos, a este le sigue la proteína con un $24.991 \%(0.084)$, ceniza $7.650 \%(0.025)$, fibra bruta 4.882 $\%(0.020)$, humedad $2.594 \%(0.022)$ y grasa $0.530 \%(0.002)$ Igualmente se observó, que los datos se mantienen dentro del rango de intervalos de confianza y de la prueba de Dixon y los análisis demostraron que el comportamiento de los datos es normal, no registra datos anómalos y no tiene tendencias a dispersarse.

Tabla 1: Composición proximal de la malanga (Colocasia esculenta Schott).

\begin{tabular}{lccccc}
\hline Parámetro & Media (\%) & $\begin{array}{c}\text { Desviación } \\
\text { estándar }\end{array}$ & Varianza & $\begin{array}{c}\text { Desviación } \\
\text { estándar } \\
\text { relativa (\%) }\end{array}$ & $\begin{array}{c}\text { Intervalo de } \\
\text { confianza }\end{array}$ \\
\hline Proteína & 24.991 & 0.084 & 0.007 & 0.339 & $24.687 \pm 25.294$ \\
Ceniza & 7.650 & 0.025 & 0.000 & 0.328 & $7.560 \pm 7.740$ \\
Fibra bruta & 4.882 & 0.020 & 0.000 & 0.420 & $4.808 \pm 4.955$ \\
Grasa & 0.530 & 0.002 & 4.333 & 0.393 & $0.522 \pm 0.537$ \\
Humedad & 2.594 & 0.022 & 0.000 & 0.859 & $2.515 \pm 2.674$ \\
Carbohidratos & 59.359 & 0.194 & 0.037 & 0.327 & $58.664 \pm 60.054$ \\
\hline
\end{tabular}

De acuerdo a la tabla 1 , el contenido de proteínas de la malanga $(24.991 \% \pm 0.084)$ es elevado, sin embargo, las proteínas de este tipo de alimentos son biológicamente incompletas (Barrera et al., 2004), porque contienen una cantidad de aminoácidos por debajo del patrón de referencia, pero teniendo en cuenta que estos alimentos son acompañados de otros en la alimentación, se suplen las deficiencias, para cumplir con el funcionamiento del organismo humano (Barrera et al., 2004). El contenido de humedad (2.594 \% \pm 0.022 ), estuvo realmente bajo, debido a que en el presente estudio el análisis se realizó en base seca; en estado fresco estos vegetales presentan aproximadamente un $68 \%$, como lo reportado por Barrera et al., 2004. El contenido de cenizas $(7.650 \% \pm 0.025)$, tiene relación con el elevado contenido de minerales presentes en los alimentos, en el caso de la malanga se ha encontrado potasio (Barrera et al., 2004), calcio, magnesio y zinc.

El contenido de fibra bruta $(4.882 \% \pm 0.020)$, forma parte integral de los carbohidratos y se puede encontrar distribuida en el tejido vegetal, dependiendo en muchos casos, del grado de maduración (Barrera et al., 2004), es por esto que se requiere identificar el periodo de cosecha de la malanga. Estos resultados son superiores a los encontrados por Eleazu et al., 2013, quienes encontraron elevadas cantidades de fibra cruda en la harina de la malanga. El contenido de grasa $(0.530 \% \pm 0.002)$ es bajo en las raíces, aunque se han encontrado contenidos de $1.75 \%$, en otras raíces y tubérculos andinos (Barrera et al., 2004). El contenido de carbohidratos de la malanga (59.359\% $\%$ 0.194), está constituido principalmente por almidón, el cual se considera de calidad y con buena de capacidad absorción de agua, lo que lo convierte en una alternativa no tradicional de almidón y puede contribuir a retomar su comercialización (Marín et al., 2016); los almidones de malanga cultivada en la región norte de Colombia, pueden tener menor contenido de amilosa, menor índice de absorción de agua, menor temperatura de gelatinización y menor índice de solubilidad en agua, en comparación con otros almidones como el del ñame y la papa, según lo reportado por Torres et al., 2013.

La tabla 2 muestra una comparación entre el contenido nutricional de la malanga con otros alimentos vegetales, estos datos fueron tomados de la Tabla de Composición química de Alimentos Colombianos (Instituto Colombiano de Bienestar Familiar 2015) y en esta se puede observar que la malanga contiene nutrientes importantes, frente a otros alimentos que se consumen con frecuencia en la región Caribe colombiana, como es el caso de la papa y la yuca. De acuerdo a esta información, la malanga supera el 
contenido de proteína y grasa de los otros vegetales, con respecto a la cantidad de calcio, también es superior a los demás, exceptuando al camote. En el contenido de carbohidratos, la malanga está por debajo del ñame y de la yuca.

Tabla 2: Comparación descriptiva entre el contenido nutricional de la malanga y otros tubérculos (Datos tomados de Instituto Colombiano de Bienestar Familiar, 2015).

\begin{tabular}{l|ccccc|}
\hline Alimento & Kcal & Proteina $(\mathrm{g})$ & Carbohidratos $(\mathrm{g})$ & Grasa $(\mathrm{g})$ & Calcio $(\mathrm{g})$ \\
\hline Malanga & 107 & 2.1 & 22.1 & 1.1 & 19.0 \\
Camote & 90 & 1.2 & 21.1 & 0.1 & 25.0 \\
Papa & 93 & 1.9 & 21.1 & 0.1 & 4.0 \\
Yuca & 153 & 0.9 & 37.1 & 0.1 & 7.0 \\
Name & 99 & 2.0 & 22.5 & 0.2 & 7.0 \\
\hline
\end{tabular}

Con relación al contenido de minerales de la malanga objeto de estudio, se determinó que el mineral presente en mayor cantidad es el magnesio $242.373 \mathrm{mg} / \mathrm{kg} \pm 0.01$, seguido del calcio $94.455 \mathrm{mg} / \mathrm{kg} \pm 0.06$, hierro 8.351 $\mathrm{mg} / \mathrm{kg} \pm 0.04$ y zinc $6.210 \mathrm{mg} / \mathrm{kg} \pm 0.03$; estos resultados obtenidos en la presente investigación, difieren de los reportados en otros estudios, como el de Mergedus et al., 2014 que reportaron los siguientes valores: 1124 $\mathrm{mg} / \mathrm{kg}$ de magnesio en los cormos centrales de la malanga, el calcio apareció en mayor proporción en la parte inferior y marginal del cormo, con valores de $892 \mathrm{mg} / \mathrm{kg}$ y $980 \mathrm{mg} / \mathrm{kg}$ respectivamente, el hierro en la parte central del cormo con $13.8 \mathrm{mg} / \mathrm{kg}$ y zinc $36.6 \mathrm{mg} / \mathrm{kg}$. Por otro lado, Arici et al., 2016, en su investigación determinaron, que entre los minerales que componían la fracción de cenizas de las harinas de la malanga, el magnesio fue el más abundante en todas las harinas y varió entre 129 y $163 \mathrm{mg} / 100 \mathrm{~g}$ de harina. Igualmente, Ezeabara et al., 2015, confirmaron la presencia de minerales como el calcio, fosforo, el sodio en mayor cantidad, y el magnesio, al que no encuentra diferencias significativas dentro de las variedades de especie de Colocasia esculenta, con una concentración que oscila entre los $68.61 \pm 0.14 \mathrm{mg} / 100 \mathrm{~g}$ y los $59.52 \pm 0.09$ $\mathrm{mg} / 100 \mathrm{~g}$. Igualmente Alinnor y Akalezi, 2010, demostraron que las muestras estudiadas de malanga son buenas fuentes de hierro y calcio. Los resultados del presente estudio permiten concluir que, el mineral con mayor presencia en la malanga (Colocasia esculenta Schott) es el magnesio, seguido del calcio. La variabilidad de minerales presentes en cada tubérculo podría estar influenciada por factores externos como la temperatura, condiciones climatológicas, tipo de suelo etc., esa diferencia puede afectar la calidad del material o muestra de análisis (Mergedus et al., 2015).

En la figura 1 se presenta el cromatograma que expresa el contenido de vitamina $\mathrm{C}$ y vitaminas del complejo B de la malanga, de la región Caribe colombiana y en la tabla 3 se amplía la información del cromatograma.

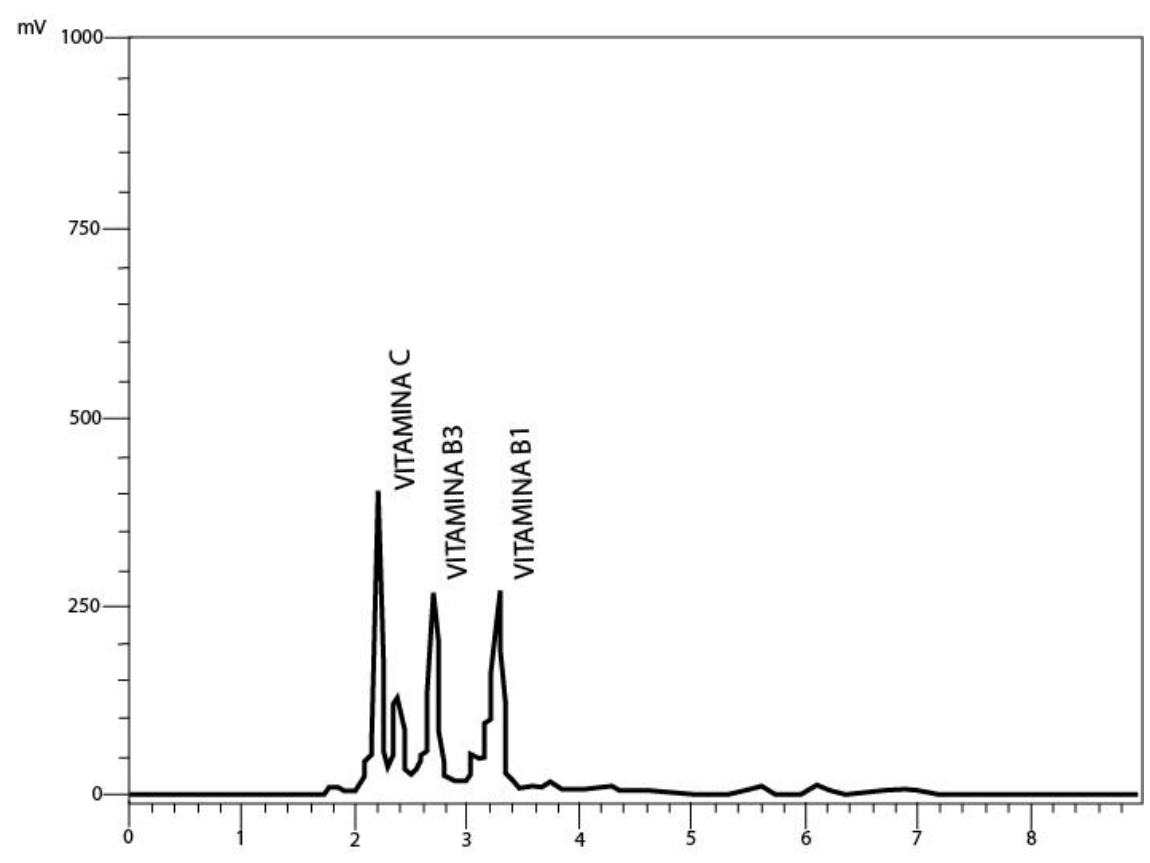

Fig. 1: Cromatograma de vitaminas de la malanga (Colocasia esculenta Schott) 
Tabla 3: Datos de cromatograma de la muestra

\begin{tabular}{|c|c|c|c|c|c|}
\hline \multicolumn{6}{|l|}{ Detector A CH1 254nm } \\
\hline Pico No & Nombre & $\begin{array}{c}\text { Tiempo de } \\
\text { retención }\end{array}$ & Área & $\begin{array}{c}\text { Percentil } \\
\text { de área }\end{array}$ & Unidades \\
\hline 1 & Vitamina C & 2.261 & 11923260.4 & 45.352 & $\%$ \\
\hline 2 & Vitamina B3 & 2.789 & 14870814.3 & 24.215 & $\%$ \\
\hline 3 & Vitamina B1 & 3.895 & 31429598.8 & 30.433 & $\%$ \\
\hline \multicolumn{7}{|c|}{ Total } & & 4428329.7 & 100 & $\%$ \\
\hline
\end{tabular}

Los resultados reportaron una media de $0.047 \mathrm{mg} / 100 \mathrm{~g}$ para vitamina B1 (tiamina), la vitamina B2 (riboflavina) y vitamina B6 (piridoxina) no fueron detectadas por el equipo en $\mathrm{mg} / 100 \mathrm{~g}$ de muestra, el contenido de vitamina B3 (niacina) fue de $0.078 \mathrm{mg} / 100 \mathrm{~g}$. Ezeabara et al., 2015, mostraron en su estudio la importancia nutricional y de salud del cultivo de malanga, reportaron que el contenido de vitamina $C$ fue de $9.28 \pm 0.02 \mathrm{mg} / 100 \mathrm{~g}$ en $C$. esculenta, estos resultados son inferiores a los obtenidos en la presente investigación. Según Ríos et al., 2017, la vitamina $C$ contenida en otros tubérculos es mayor, al compararla con la obtenida en este estudio, con $2.50 \mathrm{mg} / 100 \mathrm{~g}, 19.00 \mathrm{mg} / 100 \mathrm{~g}, 20.00 \mathrm{mg} / 100 \mathrm{~g}$ para camote, yuca, y papa respectivamente. Sin embargo, al consultar otras investigaciones, se observó que los valores manejados de esas vitaminas, que no reflejaron lectura, fueron extremadamente bajos, por ejemplo, para vitamina B2 tuvo un valor de $0.02 \mathrm{mg} / 100 \mathrm{mg}$ de muestra y para vitamina B6 lamentablemente no aparece cuantificada. Este resultado plantea que es posible encontrar en la Colocasia esculenta Schott, presencia de vitamina C y vitaminas del complejo B, a excepción de las vitaminas B2 y vitaminas B6 que no se pudieron cuantificar.

\section{CONCLUSIONES}

De acuerdo a los resultados de este estudio y de su discusión y análisis, se pueden extraer las siguientes conclusiones:

1. La malanga presenta un elevado contenido de carbohidratos y de proteínas, superior al de otros vegetales, su uso y consumo potencial puede ser prometedor, teniendo como referencia que la base de la alimentación colombiana son los carbohidratos.

2. La malanga reporta importante contenido de minerales, representados en forma de magnesio, calcio, hierro y zinc, los cuales requieren ser incluidos en la alimentación, para el cumplimiento de funciones vitales del organismo humano.

3. Vitaminas hidrosolubles como la $\mathrm{C}$ y otras vitaminas del complejo B, tales como la B1 y la B3 se encuentran en la malanga, en cantidades que pueden ser aprovechadas por la población.

4. La malanga es un vegetal que puede contribuir a mejorar la deficiencia de micronutrientres en la población, debido a que, por ser un tallo subterráneo, constituye una reserva de nutrientes en la planta.

5. Las condiciones climatológicas y el manejo del suelo en el área de muestreo, permitieron el desarrollo de un alimento con propiedades nutricionales importantes.

\section{REFERENCIAS}

Abdulrashid, M. y L. Nnabuenyi, Taro cocoyam (Colocasia esculenta) Meal as Fedd Ingredient in Poultry, doi: 10.3923/pjn2009.668.673, Pakistan Journal of Nutrition, 8(5), 668-673 (2009)

Alcantara, R.M., W.A. Hurtada y E.I. Dizon, The Nutritional Value and Phytochemical Components of Taro (Colocasia esculenta L.) Schott) Powder and its Selected Processed Foods, doi: 10.4172/2155-9600.1000207, Rev. J Ntr Food Sci, 3(3), 1-7 (2013)

Alinnor, I.J. y C.O. Akalezi, Proximate and Mineral Compositions of Dioscorea rotundata (White Yam) and Colocasia esculenta (White cocoyam), doi: 10.3923 / pjn2010.998.1001, Pakistan Journal of Nutrition, 9(10), 998-1001 (2010)

AOAC International: Official methods of Analysis of AOAC International Determination of Proteins, 18a Ed., Maryland-USA (2005)

Arici, M., R.M. Yildirim, y otros cinco autores, Physicochemical and Nutritional Properties of taro (Colocasia esculenta L. Schott) Flour as Affected by Drying Temperature and air Velocity, doi.org/10.1016/j.Iwt.2016.08.006, LWT-Food Science and Technology, 74, 434-440 (2016)

Barrera, V., C. Tapia y A. Monteros, Caracterización Físico-química, Nutricional y Funcional de Raíces y Tubérculos Andinos, En Raíces y Tubérculos Andinos: Alternativas para la conservación y uso sostenible en el Ecuador. Instituto Nacional Autónomo de Investigaciones Agropecuarias, Centro Internacional de la Papa, Agencia Suiza para el Desarrollo y la Cooperación, 91-116, Quito, Ecuador - Lima, Perú (2004) 
Eleazu, C.O., Characterization of the Natural Products in Cocoyam (Colocasia esculenta) using GC-MS, doi: 10.1080/13880209.2016.1190383, Pharmaceutical Biology, 54(12), 2880-2885 (2016)

Eleazu, C.O., M. Iroaganachi y K. C. Eleazu, Ameliorative Potentials of Cocoyam (Colocasia esculenta L.) and Unripe Plantain (Musa paradisiaca L.) on the Relative Tissue Weights of Estreptozotocin Induced Diabetic Rats, doi: 10.1155/2013/160964, Journal of Diabetic Research, 1-8 (2013)

Estrada, A., L.A. Bello y otros cuatro autores, Producción Enzimática de Maltodextrinas a Partir de Almidón de Malanga (Colocasia esculenta), doi: 1080/19476330903091300, CyTA J. Food, 7(3), 233-241 (2010)

Ezeabara, CH., C.U. Okeke y J. E.Amadi, Phytochemical, Proximate, Mineral and Vitamin Investigations of Cormels of Five Varieties of Colocasia esculenta (L.) Schott found in Anambra State, Southeastern Nigeria, Am. J. Life. Sci. Res., 3(4), 273-281 (2015)

Instituto Colombiano de Bienestar Familiar, Universidad Nacional De Colombia, Tabla de Composición de Alimentos Colombianos (TCAC), $2^{a}$ Ed., 148-149, Bogotá, Colombia (2015)

Kaushal, P., V. Kumar y H.K. Sharma, Comparative study of Physicochemical, Functional, Antinutritional and Pasting Properties of Taro (Colocasia esculenta) Rice (Oryza sativa) Flour, Pigeonpea (Cajanus cajan) Flour and Their Blends, doi:10.1016/j.Iwt.2012.02.028, Food Science and Tecnology, 48(1), 59-68 (2012)

Lewu, M.N., P.O. Adebola y A.J. Afolayan, Effect of Cooking on the Proximate Composition of the Leaves of Some Accessions of Colocasia esculenta (L) Schott in KwaZulu-Natal Province of South África, ISSN: 1684-5315, African Journal of Biotechnology, 8(8), 1619-1622 (2009)

Marín, Z., L.M. Agudelo y T. Sánchez, Caracterización del Almidón de la Malanga (Colocasia esculenta (L) Scott), doi 10.15446, Agronomía Colombiana, 34(1), 1398-1399 (2016)

Mergedus, A., J. Kristl y otros cinco autores, Variation of Mineral Composition in Different Parts of Taro (Colocasia esculenta) Corms, doi: 10.1016/j.foodchem.2014.08.025.Epub 2014 Aug 17, Food Chemistry, Rev. Bio. Agro, 170(1), 37-46 (2014)

Mongay, C., Quimiometria, Universidad de Valencia, $1^{\text {a }}$ Ed. 424, p. Valencia, España (2011)

NTC 1142: Instituto Colombiano de Normas Técnicas y Certificación-ICONTEC, Determinación del Contenido de Grasas, 1-3, Bogotá-Colombia (2000)

NTC 529: Instituto Colombiano de Normas Técnicas y Certificación-ICONTEC, Cereales y Productos Cereales. Determinación del Contenido de Humedad, 11, Bogotá-Colombia (2009)

NTC 668: Instituto Colombiano de Normas Técnicas y Certificación-ICONTEC, Alimentos y Materias Primas. Determinación de los Contenidos de Grasa y Fibra Cruda, 1-5, Bogotá-Colombia (2001)

Nwokocha, L., N.A. Aviara, Ch. Senan y P.A. Williams, A Comparative Study of Some Properties of Cassava (Manihot esculenta, Crantz) and Cocoyam (Colocasia esculenta, Linn) Starches, doi.org/10.1016/j.carbpol.2008.10.034, Carbohydrate polymers, 76(3), 362-367 (2009)

Pearson D., Técnicas de Laboratorio para el Análisis de Alimentos, Ed. Acribia, Zaragoza, España (2013)

PHARMABOOKS, USP Pharmacopeial Convention, USP 38 NF 33 (2005)

Pinzón-Bedoya, M., A.M. Cardona, Caracterización de la Cáscara de Naranja para su Uso como Material Bioadsorbente, ISSN: 0120-4211, Revista Bistúa, 6(1), 1-23 (2008)

Profamilia, Instituto Nacional de Salud, Bienestar Familiar, Ministerio De Protección Social, Encuesta Nacional de la

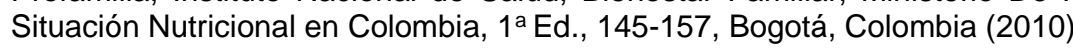

Ríos, E., H. Zelada, Determinación del Rendimiento de Glucosa por Hidrólisis Enzimática de Almidones de Yuca (Manihot esculenta), Camote (Ipomoea batatas) y Papa (Solanumtuberosum), Tesis de Grado, Facultad de Ingeniería Química, Universidad Nacional Pedro Ruíz Gallo, Lambayeque-Perú (2017)

Rodríguez-Miranda, J., J.M. Rivadeneyra-Rodríguez y otros cinco autores, Caracterización Fisicoquímica, Funcional y Contenido Fenólico de Harina de Malanga (Colocasia esculenta) Cultivada en la Región de Tuxtepec, Oaxaca, México, Ciencia y Mar, 15(13), 37-47 (2011)

Sefa-Dedeh, S. y E. K. Agyir-Sackey, Chemical Composition and the Effect of Processing on Oxalate Content of Cocoyam Xanthosomas sagittifolium and Colocasia esculenta Cormels, doi:10.1016/S0308-8146(02)00244-3, Food Chemistry, 85(4), 479-487 (2004)

Torres, A.L., P.M. Montero-Castillo y M. Durán, Propiedades Fisicoquímicas, Morfológicas y Funcionales del Almidón de Malanga (Colocasia esculenta), doi:10.22507, Rev. Lasallista de Investigación, 10(2), 52-61 (2013)

Torres-Rapelo, A.L., P.M. Montero y L.C. Julio-González, Utilización de Almidón de Malanga (Colocasia esculenta) en la Elaboración de Salchichas Tipo Frankfurt; ISSN-e: 1909-9959, ISSN: 1692-3561, Rev. Bio. Agro., 12(2), 97-106 (2014)

Viloria, H. y C. Córdova, Sistema de Producción de Ocumo Chino (Colocasia esculenta (L.) Schott) en la Parroquia Manuel Renaud del Municipio Antonio Díaz del Estado Delta Amacuro, Venezuela, Revista UDO Agrícola, 8(1), 98-106 (2008) 